\title{
Time, Causality, and Entropy
}

\author{
Amrit Šorli \\ Scientific Research Centre, Slovenija \\ amrit.sorli@bistra.si
}

Štefan Čelan

Scientific research centre Bistra, Slovenija

stefan.celan@bistra.si

\begin{abstract}
How to build an optimal model where three terms "time, causality, and entropy" will be interrelated in a most correct way? This task has a semantic and physical aspect. We give in this article the solution that is based only on elementary perception. In physics, we experience time with our senses as the duration of material change running in space. This fact is the standpoint for our model where causality is only a principle, it is not a physical actuality and entropy runs only in space and not in time. Time is merely a duration of entropy increasing and is entering the existence when measured by the observer.
\end{abstract}

Keywords: time, causality, entropy, observer

\section{Introduction}

Material changes of the universe are the indisputable reality that all physicists agree on their existence. We observe the change in the space only, never in time, and thinking that changes run in time was never observed and should be taken as an unprovable proposition. That's why we suggest we stay with the fact that changes run only in space. In this model time is the duration of changes that the observer is measuring with the clock. We have to ask: Is the duration existing before being measured? The answer is the following: we observe in the universe only the flow of changes, not their duration. Duration enters existence after the given flow of changes is compare with the other flow of changes. Clock are mechanisms that we use for a general comparison of all changes. Clocks run only in space and do not measure some general cosmological time that is non-existent, a pure theoretical unprovable proposition. Time as duration enters existence when measured by the observer. This confirms that the universe is utterly timeless, there is no trace of time in physical reality. Entropy increases only in space. There is no such a thing in the universe as a "thermodynamic arrow of time", time has no arrow and is not pointing anywhere. The flow of changes is irreversible, 
change 2 is after change 1 , and change 3 is after change 2 . We are interpreting change 1 as the cause of change 2 but this is only our interpretation, an unprovable idea. In the universe, we can only observe the flow of change that runs in space.

Universal space is time-invariant in the sense that time is not its $4^{\text {th }}$ dimension and that time does not exist in space as some real physical quantity [1]. Equipped with this understanding, the real actual relation between time, causality, and entropy become undisputable clear: time invariant universal space has a so-called "timeless configuration" [2]. The model of time reversal symmetry (T-symmetry) has no physical correspondence in the physical world. The equation of time symmetry has no physical meaning:

$$
T: t \rightarrow-t \quad(1) .
$$

There is no symmetry in time because there is no negative time $-t$. Elapsed time $t$ that is measured by the observer is not positive and is not negative, it has an absolute value:

$$
t=|t| \quad(2)[3]
$$

\section{Entropy of is increasing only in time-invariant universal space}

The increasing entropy in the universe is accepted by mainstream science, it is based on astronomical observations. We propose in this article that entropy does not increase in time because there is no physical time in the universe. In this perspective entropy and time are not physically related. "Thermodynamic arrow of time" is an empty term, "cosmological time" is an empty term.

In the universe, time is not running, only changes are running and their relative velocity depends on the variable energy density of the superfluid quantum space that is the physical origin of the universal space [4]. In principle, we can use increasing entropy as a clock, and we measure with it the duration of some other changes that run in space, but we have to understand that entropy does not increase in time and does not measure some physical time, because physical time does not exist.

\section{Discussion}

In our model causality is a concept, a model, and time when measured by the observer is also only model created by the observer. In this sense makes sense to see time as the discrete entity that measures numerical order of causality where change $\mathrm{X}$ is always before 
change $\mathrm{X}+1$. Time as the numerical order of changes is discrete, the fundamental unit of time is a Planck time. Every elapsed time can be seen as a sum of Planck times:

$$
t=t_{P 1}+t_{P 2}+\cdots+t_{P N}=\sum_{i=1}^{N} t_{P i} \quad \text { (3) [5]. }
$$

That time is a discrete entity is also suggested by the recent research: With the introduction of causality introduced here, time can only be reconstructed as a discrete entity also yielding other consequences from entropy to the evolution of the universe and many other findings, and thus opening more avenues to be studied. We, therefore, invite the reader to participate in this journey [6].

In other recent research is suggested that the flow of change and the flow of quanta embodies the flow of time: "It is difficult to break the habit of thinking that time is not a dimension. Still, there is no universal axis along which to organize all events since events occur in relation to an observer. Time is relative. The passage of time that I experience matters to me, the one you sense matters to you. Greenwich Mean Time (GMT) serves to synchronize events globally, but it is just a local convention in the universe. For example, what took place on our neighboring star, Proxima Centauri, about four years ago, is visible only here today. Time is not just what can be timed, so to speak, operational comparison. A running clock is also a system in a state of imbalance. The ticking is a series of events targeting balance; the flow of quanta embodies the flow of time" [7]. In our model, this is true only in the sense that so called "fundamental time" is the numerical order of this flow of quanta that runs in time-invariant universal space. The fundamental unit of the numerical order of quanta flows is a Planck time. Duration of quanta flow that we call "emergent time" does not exist on its own. Its existence requires measurement from the side of the observer $[5]$.

Our model of time resolves the puzzle of the Einstein-Podolsky-Rosen experiment where the transfer of information between two entangled particles is immediate which is not in accord with the theory of relativity: "In modern language, the Einstein-Podolsky-Rosen paradox, in its most elementary form, is concerned with two entangled particles with opposite spin. We do not know the spins of the individual particles, only that they were both created at time T1 with total spin equal to zero; once we measure the spin of one of the particles at time T2, we can immediately know the spin of the other particle, even if they are separated by a very large distance. So it appears as though the influence of the measurement on the first particle instantaneously reaches the second particle, which contradicts the principles of the 
theory of relativity since such an influence should not be able to travel faster than light" [8]. In the universe, there is no time $\mathrm{T} 1$ and some other time $\mathrm{T} 2$, changes run in time-invariant space. Fundamental time is the sequential numerical order of events which run in timeinvariant universal space. When event $\mathrm{X}+1$ enters existence, event $\mathrm{X}$ is not in existence anymore. When event $\mathrm{X}+2$ enters existence, the event $\mathrm{X}+1$ is not in existence anymore. The only real existent event is the one that we perceive with senses and measure with instruments. Fundamental time runs without being observed by the observer. But without the observation and measurement from the side of the observer, the fundamental time has no meaning of its own. The numerical order of events (events are following one after another) gets the meaning when being observed. When we measure it with the clock, we get the duration. In our model of relativity, there is no contradiction. The time-invariant universal space is the direct medium of entanglement EPR-type. An entanglement is an event that has no numerical order and so no duration [4].

In our model, the universe is timeless in the sense that it does not run in some physical time, it runs only in the time-invariant space. Past and future do not exist eternally as it is proposed in a block universe: "Timelessness", as such, is not a new idea. It was proposed in a specific form by Albert Einstein. In a block universe, all of space and time is claimed to exist eternally" [9]. In our model, the universe is timeless in the sense that Eternity is not infinitely back into the past and infinitely forward into the future, eternity is NOW. Humans, we experience the time-invariant property of the universal space as NOW. Universal changes run in NOW. What we observe in NOW is the only real universe that has physical existence. Albert Einstein has said: "For us believing physicists the distinction between past, present, and future only has the meaning of an illusion, though a persistent one" [10]. Yet even the great Einstein despaired of understanding the flow of time and the meaning of now. Einstein's quandary was described by Rudolf Carnap: "Einstein said the problem of the Now worried him seriously. He explained that the experience of the Now means something special for man, something essentially different from the past and the future, but that this important difference does not and cannot occur within physics. That this experience cannot be grasped by science seemed to him a matter of painful but inevitable resignation. So he concluded that there is something essential about the Now which is just outside the realm of science" [11]. We brought NOW into science with the model of the time-invariant universal space where time as duration enters existence when measured by the observer. 


\section{Conclusions}

Causality, where cause and effect are existing in some physical time, is a false idea because there is no physical time. Causality is merely an abstraction, cause, and effect are not based on the elementary perception, they belong to philosophy, they are not essential terms of physics. An increase of entropy has nothing to do with the flow of time as there is no physical flow of time in the universe. The entropy of a given system is increasing in the time-invariant universal space. Also, the flow of time in which change run is a philosophical concept that misleads physics on the wrong path. Changes run only in space. Time is their duration and enters existence when being measured by the observer. No measurement means no time. Since the introduction of quantum superposition, we know that the observer's observation and measurement are an essential part of physics. We show in this article that the observer's observation and measurement also create time. This discovery could be a paradigm shift of 21-century: physics is alive, we physicists are its constitutive part.

\section{References:}

1. Šorli, A.S. \& Čelan Š., Time-Invariant Superfluid Quantum Space as the Unified Field Theory, RAPS, Vol. 4, No. 3 (2020) 2050007 , https://doi.org/10.1142/S2424942420500073 (2021)

2. Gomes, H. Timeless Configuration Space and the Emergence of Classical Behavior. Found Phys 48, 668-715 (2018). https://doi.org/10.1007/s 10701-018-0172$\underline{1}$

3. Šorli A. and Čelan Š., Inconsistency of Time-symmetry Model, accepted in publication in Physics Essays

4. Šorli A and Čelan Š., Advances of Relativity Theory, Physics Essays, Vol 34: Pages 201-210 (2021) http://dx.doi.org/10.4006/0836-1398-34.2.201

5. Fiscaletti, D., Sorli, A. Perspectives of the Numerical Order of Material Changes in Timeless Approaches in Physics. Found Phys 45, 105-133 (2015).

https://doi.org/10.1007/s10701-014-9840-y

6. Riek, R.; Chatterjee, A. Causality in Discrete Time Physics Derived from Maupertuis Reduced Action Principle. Entropy 2021, 23, 1212.

https://doi.org/10.3390/e23091212

7. Annila, A. The Matter of Time. Entropy 2021, 23, 943. https://doi.org/10.3390/e23080943

8. Tamm, M. Is Causality a Necessary Tool for Understanding Our Universe, or Is It a Part of the Problem? Entropy 2021, 23, 886. https://doi.org/10.3390/e23070886 
9. Thomsen, K. Timelessness Strictly inside the Quantum Realm. Entropy 2021, 23, 772. https://doi.org/10.3390/e23060772

10. Bracco C. Einstein and Besso: From Zürich to Milano. https://arxiv.org/ftp/arxiv/papers/1412/1412.6981.pdf

11. Muller R. NOW: The physics of Time, W. W. Norton \& Company; Illustrated edition (September 20, 2016) https://www.npr.org/sections/13.7/2016/09/27/495608371/nowand-the-physics-of-time 